\title{
CDMA Overlay Situations for Microcellular Mobile Communications
}

\author{
Jiangzhou Wang, Senior Member, IEEE, and Laurence B. Milstein, Fellow, IEEE
}

\begin{abstract}
Direct sequence code division multiple access communications is a promising approach to cellular mobile communications, which operates in an environment characterized by multipath Rician fading. In this paper, the CDMA network is assumed to share common spectrum with a narrowband microwave user. Because of the presence of the narrowband waveform, an interference suppression filter at each CDMA receiver is employed to reject the narrowband interference. The problem of interference from adjacent cells is also considered. Average power control is assumed to combat the near/far problem, and multipath diversity, in conjunction with simple interleaved channel coding, is considered for Improving the performance of the CDMA system.
\end{abstract}

\section{INTRODUCTION}

Code division multiple access (CDMA) is becoming a very attractive technique for personal communications networks (PCN) and microcellular mobile communications [17]. One reason is that the CDMA code sequences inherently combat multipath which is a major concern in mobile radio communications. Also, since the CDMA signals spread their power over a large bandwidth, the effect of such a transmission on a narrowband receiver is often just an imperceptible rise in its noise level. Hence, it may be possible to overlay a CDMA system on an existing set of microwave narrowband users without adversely affecting either. Moreover, because of the broadband noise-like character of direct sequence (DS) CDMA signals, it is possible to use newly developed signal processing techniques to perform narrowband interference suppression [14]. This will clearly reduce the narrowband interference for CDMA users, but it will also help the narrowband users, since it is then possible to operate the CDMA transmissions at a reduced power level.

As is well known, microcellular mobile radio can increase the capacity of future cellular systems. The chief advantages of the microcells are the enhanced spectrum efficiency and low power consumption of the handsets. Microcells, as the name indicates, are relatively much smaller in size $(0.2-1 \mathrm{~km}$

This paper was approved by James S. Lehner, the Editor for Modulation \& Signal Design of the IEEE Communications Society. Manuscript received: December 9, 1991; revised: January 11, 1993.

This work was supported by the Office of Naval Research under Grant N00014-91-J-1234 and the National Science Foundation Industry/University Cooperative Research Center on Ultra-High Speed Integrated Circuits and Systems at the University of Califomia, San Diego.

Jiangzhou Wang is with Digital Communications Division, Rockwell Corporation, Newport Beach, CA 92658-8902

Laurence B. Milstein is with the Department of Electrical \& Computer Engincering, University of Califomia, San Diego, La Jolla, CA 92093

IEEE Log Number 9410093 radius) than the conventional cells ( $1-10 \mathrm{~km}$ radius). Also, microcells operate at lower power and have their antennas at streetlamp elevations. In [3], it is shown that in multipath models the propagation path (per path) loss exponent for close-in cells can be between two and three in microcellular radio, instead of four in conventional cellular and terrestrial radio. Also, the propagation measurements in [4] characterize the microcellular environments as a multipath Rician (rather than Rayleigh) fading channel.

Because of the presence of the narrowband waveform, a suppression filter at each CDMA receiver is employed to reject the interference. The problem of interference from adjacent celis is considered. Also, diversity is used in conjunction with block interleaved coding for improving the BER performance. The outline of the paper is as follows. The system and channel models are described in Section II. Section III derives an approximation to the bit error rate (BER) performance of the DS-CDMA overlay system, and this is followed by both representative numerical results and a discussion of these results in Section IV. Finally, conclusions are presented in Section V.

\section{SYSTEM AND CHANNEL MODELS}

\section{A. Transmitter Model}

The transmitter model of a DS system with interleaved coding and PSK modulation consists of a channel encoder, an interleaver and a DS spreader (or modulator). The transmitted signal of the $k$ th user in the CDMA system takes the form

$$
S_{k}(t)=\operatorname{Re}\left\{\sqrt{2 P_{k}} b_{k}(t) a_{k}(t) \exp \left[j\left(2 \pi f_{0} t+\theta_{k}\right)\right]\right\},
$$

where $\operatorname{Re}\{\cdot\}$ stands for real part, $f_{0}$ denotes the carrier frequency, which is common to all users, and $P_{k}$ and $\theta_{k}$ are the transmitted power and phase of the kth user, respectively. The symbol $b_{k}(t)$ is the $k$ th interleaved and coded information bit; the bit rate $R_{b}=1 / T_{b}$, where $T_{b}$ denotes the duration of a coded symbol. The information bit rate $R_{i}$ (or the rate of source bits) and information bit duration $T_{i}$ are given by $R_{i}=1 / T_{i}=(m / n) R_{b}$ and $T_{i}=T_{b} /(m / n)$, respectively, wherc $m / n$ is the rate of the code. The $\lambda$ th square pulse of $b_{k}(t)$ has amplitude $b_{k}^{(\lambda)}$, taking values from $\{+1,-1\}$. The random signature sequence waveform $a_{k}(t)$ has a chip rate $1 / T_{c}$, and the $j$ th chip of $a_{k}(t)$ is denoted by $a_{j}^{(k)}$. We assume that there are $N$ chips (processing gain) of the random signature sequence in each coded bit $\left(T_{b}=N T_{c}\right)$. Therefore, the spread spectrum bandwidth $\left(B_{s}\right)$ is approximately given by 


$$
B_{s}=2 T_{c}^{-1}=2 N\left(T_{i} m / n\right)^{-1} \text {. }
$$

\section{B. Channel Model}

The mobile radio channel can be modeled as a discrete multipath fading channel. "Discrete multipath" means that the received signal is the sum of a finite number of delayed versions of the transmitted signal, whereas "fading" refers to the fact that, for a given transmitted power, the received power fluctuates in a random fashion. Because of the multipath nature of the channel, the fading is frequency-selective.

The propagation measurements in [4] characterize the microcellular environments as a multipath Rician fading channel, where the received signal consists of several specular components plus several Rayleigh-fading components. The multipath Rician fading channel between the $k$ th user and the receiver of interest (namely the receiver in the base station of what we refer to as the first cell) is modeled by the complex lowpass equivalent impulse response

$h_{k}(t)=\frac{1}{\left(d_{1, k}\right)^{\gamma / 2}} \sum_{l=1}^{L}\left[A_{k l} \exp \left(j \eta_{k l}\right)+\beta_{k l} \exp \left(j \mu_{k l}\right)\right] \delta\left(t-\tau_{k l}\right)$,

where $d_{1, k}\left(d_{1, k} \neq 0\right)$ is the distance between the $k$ th user and the first cell base station, and $\gamma$ is the propagation path loss exponent. In (3), $A_{k l}\left(0 \leq A_{k l} \leq 1\right)$ and $\eta_{k l}$ are gain and phase of the specular component of the lth path from the kth user, respectively; they are assumed to be deterministic and nearly constant over the duration of at least one bit. The random gain $\beta_{k t}$ and random phase $\mu_{k t}$ of the fading component of the lth path of the kth user have a Rayleigh distribution with $E\left[\beta_{k l}^{2}\right]=2 \rho_{k l}$, and a uniform distribution in $[0,2 \pi]$, respectively. The path delay, $\tau_{k l}$, is uniformly distributed in $\left[0, T_{b}\right]$. We assume that there are $L$ paths associated with each user. The gains, delays and phases of different paths and/or of different users are all statistically independent. We define the quantity $H_{k l}$, as the ratio of the specular component power to the Rayleigh fading power. That is,

$$
H_{k l} \equiv A_{k l}^{2} / E\left[\beta_{k l]}^{2}\right]=A_{k l}^{2} /\left(2 \rho_{k l}\right) \text {. }
$$

The microcellular (outdoor) channel measurements from [4] show that Rician distributions occur with $H_{k}$ values ranging from $7 \mathrm{~dB}$ to $12 \mathrm{~dB}$, instead of $2 \mathrm{~dB}$ to $7 \mathrm{~dB}$ for an indoor radio channel [7].

The interference in the channel is assumed to be a nonfading narrowband BPSK signal, and is given by

$$
J(t)=\operatorname{Re}\left\{\sqrt{2 J} d(t) \exp \left[j\left[2 \pi\left(f_{0}+\Delta\right) t+\Theta\right]\right]\right\},
$$

where $\Delta$ stands for the offset of the interference carrier frequency from the carrier frequency $\left(f_{0}\right)$ of the CDMA signals. The parameters $J$ and $\Theta$ denote the received interference power and phase, respectively. The information sequence $d(t)$ has the bit rate $T_{j}^{-1}$, where $T_{j}$ denotes the duration of one bit. Therefore, the interference bandwidth is approximately $B_{j}=2 T_{j}^{-1}$ (we assume $B_{j}<B_{s}$ ). The ratio $(p)$ of the interference bandwidth to the spread spectrum bandwidth and the ratio $(q)$ of the offset of the interference carrier frequency to half of the spread spectrum bandwidth are defined as, respectively,

$$
p=B_{j} / B_{s}=T_{c} / T_{j}
$$

and

$$
q=\Delta\left(B_{s} / 2\right)=\Delta T_{c} .
$$

Therefore, the received signal $r(t)$ can be represented as

$$
\begin{aligned}
r(t) & =\operatorname{Re}\left\{\sqrt{2 P} \sum_{k=1}^{C K} \sqrt{\varepsilon\left(\gamma, c_{k}, k\right)} \sum_{l=1}^{L}\left[A_{k l} \exp \left(j \phi_{k l}\right)+\beta_{k l} \exp \left(j \psi_{k l}\right)\right]\right. \\
& \left.\cdot b_{k}\left(t-\tau_{k l}\right) a_{k}\left(t-\tau_{k l}\right) \exp \left(j 2 \pi f_{0} t\right)\right\}+J(t)+n(t)
\end{aligned}
$$

where $n(t)$ is an AWGN process with two-sided power spectral density $\quad N_{0} / 2, \phi_{k l} \equiv \theta_{k}+\eta_{k l}-2 \pi f_{0} \tau_{k l}, \quad \psi_{k l} \equiv \theta_{k}$ $+\mu_{k l}-2 \pi f_{0} \tau_{k l}, \mathrm{C}$ stands for the number of cells, each one containing $K$ users, and $c_{k}$ denotes the $c_{k}$ th cell (the integer portion of $\left.1+(k-1) / K, c_{k}=1, \cdots, C\right)$. The first cell $\left(c_{k}=1\right)$ is defined as the cell of interest, and $P$ and $\varepsilon\left(\gamma, c_{k}, k\right)$ are defined as

$$
P=P_{k} /\left(d_{c_{k}, k}\right)^{\gamma}=\text { constant }
$$

and

$$
\varepsilon\left(\gamma, c_{k}, k\right)=\left(d_{c_{k}, k} / d_{1, k}\right)^{\gamma},
$$

respectively, where $d_{c_{r}, k}$ is the distance of the $k$ th mobile user to its own base station (the $c_{k}$ th cell), and $d_{c_{2}, k} \neq 0$. The constant $P$ means each base station provides adaptive power control to all $K$ users of its own cell so that the received signals from its cell have approximately equal power to avoid the near/far problem.

\section{Receiver Model}

As shown in Fig. 1(a), the receiver contains a suppression filter, a bank of bandpass (BP) matched filters, a bank of $U$ parallel PSK demodulators and diversity circuits a hard decision device, a deinterleaver and a hard decision decoder. A detailed model of the PSK demodulators and diversity circuit is shown in Fig. 1(b).

Notice that, for simplicity, we have ignored any bandpass filtering at the front-end of the suppression filter. We take the suppression filter to be a double-sided Wiener filter with $M$ taps on each side. Its impulse response is $\sum_{m=-M}^{M} \alpha_{m} \delta\left(t-m T_{c}\right)$, where $\alpha_{0}=1$ and $\alpha_{m}=\alpha_{-m}$. The resulting suppression filter output is given by

$$
r_{f}(t)=\sum_{m=-M}^{M} \alpha_{m} r\left(t-m T_{c}\right),
$$

where $f_{0} T_{c}$ is taken to be an integer.

The signal $r_{f}(t)$ enters a bank of BP matched filters, whose impulse responses are matched to consecutive $T_{b}$ second segments of $2 a_{i}(t) \cos \left(2 \pi f_{0} t\right) P_{T_{0}}\left(t-v T_{b}\right)$, where $P_{T_{b}}(t)=1$ for $0 \leq t<T_{b}$ and is 0 otherwise, $v=1, \cdots, V$, 


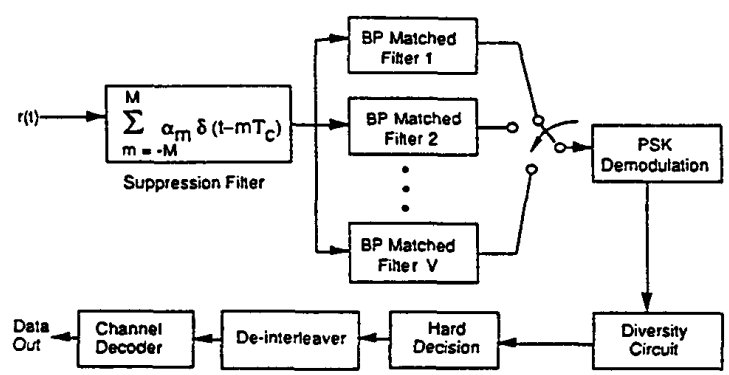

(a)

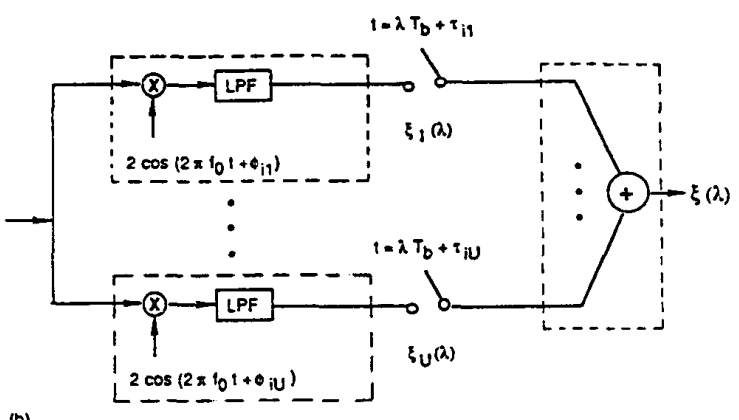

(b)

Fig. 1. (a) Receiver model of a CDMA overlay system. (b) A detailed model of the PSK demodulators and the diversity circuit.

and $V$ is the number of matched filters. Also it is assumed that the $i$ th user of the first cell is the reference user. During each bit duration, the BP signal at the output of the correct matched filter contains $2 M+1$ narrow pulses, each of width $2 T_{c}$, centered at instants $\lambda T_{b}+\tau_{i l}+m T_{c}$ for $m=-M, \cdots, 0, \cdots, M$, which are caused by the $2 M+1$ taps of the suppression filter and the $l$ th $(l=1,2, \cdots, L)$ path of the reference user. Of the $2 M+1$ peaks, the middle peak $(\mathrm{m}=0)$ is the largest (or main) peak, due to the zero-th tap of the suppression filter, whereas sidelobe peaks (or earlier and later peaks) are due to the taps excluding the zero-th tap. We assume that $\left|\tau_{i l}-\tau_{i i}\right| \geq(2 M+1) T_{c}$ for $\hat{l}=1, \cdots, L, \hat{l} \neq l$ (see Fig. 2), since if $\left|\tau_{i l}-\tau_{i l}\right|<(2 M+1) T_{c}$, the overlay of the outputs due to different paths might preclude the paths from being resolvable. We further assume that the receiver only uses the combination of the main peaks of the resolvable paths of the reference user to form the diversity. Paths from non-reference users do not give rise to regular peaks, because their spreading sequences are different from $a_{i}(t)$.

In any given symbol interval, the output of the appropriate BP matched filter enters $U$ conventional PSK demodulators, $1 \leq U \leq L$, (Fig. 1(b)), corresponding to $U$ different resolvable paths of the reference user. The coherent receiver makes use of carrier reference signals $\left\{2 \cos \left(2 \pi f_{0} t+\phi_{i l}\right)\right\}$, which are in phase with the specular components of the different signals from the reference user. These carrier reference signals can be derived from the BP matched filter out-
BP matched filter output envelope

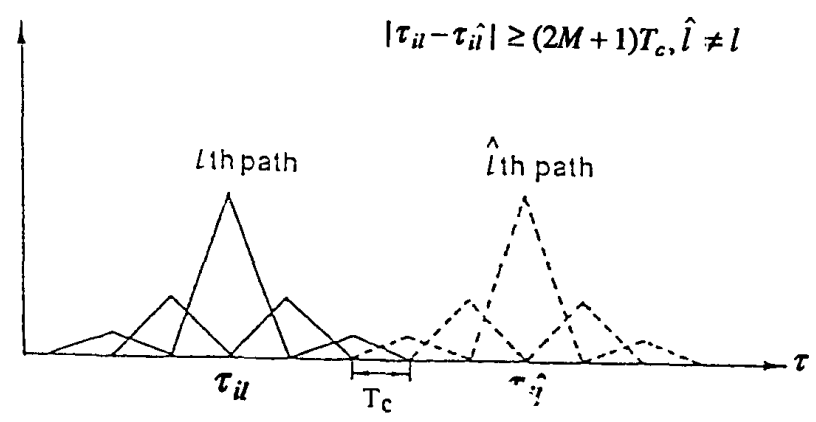

Fig. 2. The matched filter output envelope in CDMA overlay situation with suppression filters $(2 \mathrm{M}+1=5)$ over a multipath channel.

puts by means of carrier synchronization circuits (one circuit per considered path). The output of the $l$ th, $1 \leq l \leq U$, demodulator is a lowpass signal, which has $2 M+1$ peaks at the instants $\lambda T_{b}+\tau_{i l}+m T_{c}, \quad m=-M, \cdots, 0, \cdots, M$. The desired component of the lowpass output signal (i.e., the main peak, $m=0$ ) of the $l$ th demodulator is sampled at the instant $t=\lambda T_{b}+\tau_{i l}$, and this gives rise to the random variable $\xi_{l}(\lambda)$. Note that the time difference between any two samples is at least equal to $(2 M+1) T_{c}$. The $\mathrm{U}$ random variables, $\xi_{l}(\lambda), l=1, \cdots, U$, are directly summed to form the decision variable $\xi(\lambda)$.

\section{SYSTEM PERFORMANCE}

The $l$ th random variable, $\xi_{l}(\lambda)$, for detection can be written as

$$
\xi_{l}(\lambda)=\int_{\lambda T_{b}+\tau_{i l}}^{(\lambda+1) T_{b}+\tau_{i j}} r_{f}(t) a_{i}\left(t-\tau_{i l}\right)
$$

where $2 \cos \left(2 \pi f_{0} t+\phi_{i l}\right)$ is the recovered carrier of the specular component of the $l$ th resolvable path of the reference user and $a_{i}\left(t-\tau_{i l}\right)$ is the random spreading sequence of the $l$ th path of the reference user. Since the high frequency terms are removed by the lowpass filter following the mixer of the demodulator, the above expression reduces to

$$
\begin{aligned}
& \xi_{l}(\lambda)=\sqrt{2 P} A_{i l} T_{b} b_{i}^{(\lambda)}+D_{l}(\lambda)+F_{l}(\lambda)+N_{l}(\lambda)+J_{l}(\lambda)+\sum_{l=1}^{L} I_{i, l, i}
\end{aligned}
$$

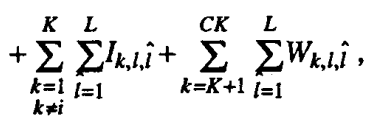

where the terms on the right hand side of (13) are described in detail below.

--- The first term is the desired signal, corresponding to the specular component of the $l$ th path of the reference user and the zero-th tap of the suppression filter. The power of this term equals

$$
S_{c}=2 P A^{2} T_{b}^{2},
$$


where it is assumed that $A_{i l}=A$ for all $i$ and $l$. For most of the remaining terms, we are interested in their conditional variances, conditioned on $b_{i}^{(\lambda)}$ and $a_{i}(t)$. Let us consider them one at a time.

-- $D_{l}(\lambda)$ is an interference term due to the fading component of the lth path of the reference user and the zero-th tap of the suppression filter, and is given by

$$
D_{l}(\lambda)=\sqrt{2 P} \beta_{i l} T_{b} b_{i}^{(\lambda)} \cos \left(\mu_{i l}-\eta_{i l}\right) \text {. }
$$

Note that this term is a conditional zero-mean Gaussian random variable with variance $2 P \rho T_{b}^{2}$, where it is assumed that $\rho_{i l}=E\left(\beta_{i l}^{2}\right) / 2=\rho$ for all $i$ and $l$.

-- $F_{l}(\lambda)$ is a self interference term due to the $l$ th path of the reference user which is caused by the non-reference taps of the suppression filter; it is given by

$$
\begin{aligned}
F_{l}(\lambda)= & \sqrt{2 P}\left[A_{i l}+\beta_{i l} \cos \left(\mu_{i l}-\eta_{i l}\right)\right] \sum_{\substack{m=-M \\
m \neq 0}}^{M} \alpha_{m} \\
& \quad \cdot \int_{\lambda T_{b}+\tau_{i l}}^{(\lambda+1) T_{b}+\tau_{u}} b_{i}\left(t-m T_{c}-\tau_{i l}\right) a_{i}\left(t-m T_{c}-\tau_{i l}\right) a_{i}\left(t-\tau_{i l}\right) d t .
\end{aligned}
$$

For relatively small narrowband interference bandwidths (relative to the spread bandwidth of the CDMA signals), this term does not have a significant effect, and thus it will be ignored in what follows.

-- $N(\lambda)$ is due to the thermal noise; its conditional variance equals $N_{0} T_{b} \sum_{m=-M}^{M} \alpha_{m}^{2}$.

--- $J_{l}(\lambda)$ is due to the BPSK narrowband interference, and is given by

$$
\begin{array}{r}
J_{l}(\lambda)=\sum_{m=-M}^{M} \alpha_{m} \int_{\lambda T_{b}+\tau_{a l}}^{(\lambda+1) T_{b}+\tau_{i l}} \sqrt{2 J} d\left(t-m T_{c}\right) \\
\cdot \cos \left[2 \pi \Delta\left(t-m T_{c}\right)+\hat{\Theta}\right] a_{i}\left(t-\tau_{i l}\right) d t,
\end{array}
$$

where $\hat{\Theta} \equiv \Theta+\phi_{i l}$ and $f_{0} T_{c}$ is assumed to be an integer. This term is very significant, and will be treated separately.

-- $I_{i, l, l}(\hat{l} \neq l)$ is a multipath interference term due to the $\hat{l}_{\text {th }}$ path of the reference user and is given by

$$
\begin{aligned}
& I_{i, l, \hat{l}}=\sqrt{2 P}\left[A_{i \hat{i}} \cos \left(\phi_{i \hat{l}}-\phi_{i l}\right)+\beta_{i \hat{l}} \cos \left(\psi_{i \hat{l}}-\phi_{i l}\right)\right] \sum_{m=-M}^{M} \alpha_{m} \\
& \quad \cdot(\lambda+1) T_{b}+\tau_{i l} \\
& \lambda \int_{t+\tau_{\sharp}} b_{i}\left(t-\tau_{i \hat{l}}-m T_{c}\right) a_{i}\left(t-\tau_{i \hat{l}}-m T_{c}\right) a_{i}\left(t-\tau_{i l}\right) d t
\end{aligned}
$$

For a large number of CDMA users (i.e., for $K \gg 1$ ), the effect of the multipath of the desired user is very small, since it roughly acts as one additional user. However, its inclusion in the analysis greatly complicates that analysis, and so, as was the case with $F_{l}(\lambda)$, it will be ignored.

--- $I_{k, l, \hat{l}}(k \neq i)$ is a multi-access interference term due to the $l$ th path of the $k$ th user in the first cell (i.e., $\mathrm{c}=1$ and $\mathrm{k}=1,2, \cdots$, $\mathrm{K} ; k \neq i)$ and is given by

$$
I_{k, l, \hat{l}}=\sqrt{2 P}\left[A_{k \hat{l}} \cos \left(\phi_{k \hat{l}}-\phi_{i l}\right)+\beta_{k \hat{l}} \cos \left(\psi_{k \hat{l}}-\phi_{i l}\right)\right] \sum_{m=-M}^{M} \alpha_{m}
$$

$$
\stackrel{\int_{i}+\tau_{k}}{(\lambda+1) T_{b}+\tau_{i l}} b_{k}\left(t-\tau_{k \hat{l}}-m T_{c}\right) a_{k}\left(t-\tau_{k \hat{l}}-m T_{c}\right) a_{i}\left(t-\tau_{i l}\right) d t .
$$

The random variable $I_{k, l, \hat{l}}$ has a conditional variance equal to approximately $\sigma_{0}^{2}$, where

$\sigma_{0}^{2}=\frac{P\left(A^{2}+2 \rho\right)}{3 N}\left[2 \sum_{m=-M}^{M} \alpha_{m}^{2}+\sum_{m=-M}^{M} \alpha_{m} \alpha_{m+1}\right] T_{b}^{2}$,

where $\alpha_{m}=0$ for $|m|>M$. Therefore, the conditional variance of the total multi-access interference term in (13) equals $(K-1) L \sigma_{0}^{2}$.

... $W_{k, l, i}$ is the interference term due to the $\hat{l}_{\text {th }}$ path of the $k$ th user in the $c_{k}$ th adjacent cell $\left(\mathrm{k}=\mathrm{K}+1, \cdots, \mathrm{CK}\right.$ and $\left.c_{k} \neq 1\right)$, given by

$$
W_{k, l, \hat{l}}=\sqrt{\varepsilon\left(\gamma, c_{k}, k\right)} I_{k, l, \hat{l}},
$$

where $I_{k, l, \hat{l}}$ is given by (19). $W_{k, l, \hat{l}}$ has the conditional variance, conditioned on $\varepsilon\left(\gamma, c_{k}, k\right)$, given by

$$
\left.E\left[W_{k, l, i}^{2}\right]\right|_{\varepsilon\left(\gamma, c_{k}, k\right)}=\varepsilon\left(\gamma, c_{k}, k\right) \sigma_{0}^{2} .
$$

An exact description of the adjacent-cell interference in the hexagonal cellular model, shown in Fig. 3(a), is difficult, because the value of (22) depends on the position of the kth mobile user in the $c_{k}$ th cell $\left(c_{k} \neq 1\right)$. In order to avoid the dependence on the position, we average the result of (22) over the area of $c_{k}$ th cell. Also, we approximate the hexagonal cell with a circular cell of equal area as in [5] (a hexagonal cell inscribed in a circular cell of radius $1.035 r$ is equal in area to a circular cell of radius r), as shown in Fig. 3(b). (Note that there appears to be an error in the calculation of the radius of the circular cell in [5].) The factor $\varepsilon\left(\gamma, c_{k}, k\right)$, for $c_{k}=2, \cdots, C$, is given by

$$
\varepsilon\left(\gamma, c_{k}, k\right)=\left[\frac{x^{2}}{(x \cos \theta)^{2}+(y+x \sin \theta)^{2}}\right]^{\gamma / 2},
$$

where $\mathrm{y}$ is the distance between the $c_{k}$ th cell base station and the first cell base station. As shown in Fig. 3(a), the distance between any cell base station in the first layer of cells and the first cell base station is identical ( $y=\sqrt{3} * 1.035 r=1.793 r$ ), where $r$ is the radius of the circular cell, and the first layer contains six cells. The second layer of cells contains twelve cells, of which six have the distance $y=2 \sqrt{3} * 1.035 r=3.586 r$ from the first cell base station and the other six have the distance $y=3^{*} 1.035 r=3.105 r$ from the first cell base station. Finally, the third layer of cells contains eighteen cells, of which six have the distance $y=3 \sqrt{3} * 1.035 r=5.379 r$ and the other twelve have the distance $y=\sqrt{4.5^{2}+0.75^{2}} * 1.035 r \approx 4.74 r$ from the first cell base station. Referring to Fig. 3(b), the average of (23) over the $c_{k}$ th cell area is given by

$$
\begin{aligned}
\varepsilon\left(\gamma, c_{k}\right) & =E\left[\varepsilon\left(\gamma, c_{k}, k\right)\right] \\
& =\frac{1}{\pi r^{2}} \int_{0}^{r} \int_{0}^{2 \pi}\left[\frac{x^{2}}{(x \cos \theta)^{2}+(y+x \sin \theta)^{2}}\right]^{\gamma / 2} x d x d \theta \\
& =\frac{1}{\pi} \int_{0}^{1} \int_{0}^{2 \pi}\left[\frac{\hat{x}^{2}}{(\hat{x} \cos \theta)^{2}+(y / r+\hat{x} \sin \theta)^{2}}\right]^{\gamma / 2} \hat{x} d \hat{x} d \theta .
\end{aligned}
$$




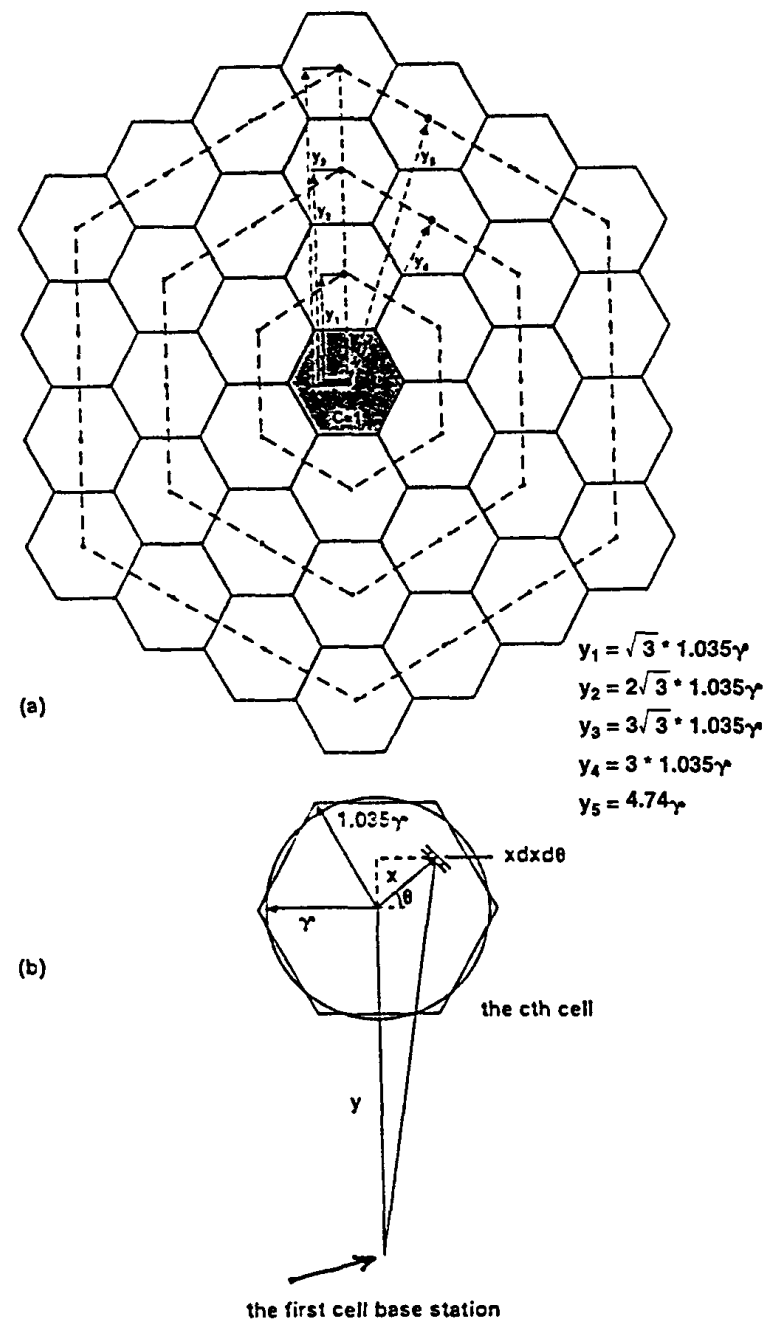

Fig. 3. (a) The microcellular model. (b) The hexagonal cell approximated by a circular cell of equal area.
Table I shows the result of (24) for $\gamma=2,3,4$ and for the various values of $y$. Therefore, the conditional variance of the total adjacent-cell interference involved with $G$ layers of cells is approximated by

$$
\sigma^{2}=\zeta(\gamma) K L \sigma_{0}^{2} .
$$

In (25), the factor $\zeta(\gamma)$ is due to all adjacent cells and is given by

$$
\zeta(\gamma)=\sum_{j=1}^{G} \zeta_{j}(\gamma),
$$

where $\zeta_{j}(\gamma)$ is defined as

$$
\zeta_{j}(\gamma)=\sum_{c_{k} \in s_{j}} \varepsilon\left(\gamma, c_{k}\right),
$$

and where $s_{j}$ is the set of all cells in the jth layer.

Note that the factors $\varepsilon\left(\gamma, c_{k}\right)$, given by (24), are independent of cell size because we normalize distance to the cell radius. Thus, the total factor $\zeta(\gamma)$, given by (26), is due only to the number of layers of cells (or, equivalently, the number of cells).

\section{A. Average Probability of Error.}

To obtain an approximation to the average probability of error, we will use the general approach of [8] - [11], although the presence of the narrowband interference complicates the derivation. Before proceeding, let us first simplify the notation. The final test statistic is given by

$$
\psi(\lambda)=\sum_{l=1}^{U} \xi_{l}(\lambda),
$$

\begin{tabular}{|c|c|c|c|c|c|c|c|c|c|c|c|}
\hline \multirow{3}{*}{$\gamma$} & \multicolumn{2}{|c|}{$\begin{array}{l}\text { First Layer } \\
\text { of cclls }\end{array}$} & \multicolumn{3}{|c|}{$\begin{array}{l}\text { Second Layer } \\
\text { of cells }\end{array}$} & \multicolumn{3}{|c|}{$\begin{array}{l}\text { Third Layer } \\
\text { of cclls }\end{array}$} & \multicolumn{3}{|c|}{$\zeta(\gamma)$} \\
\hline & \multirow{2}{*}{$\begin{array}{l}\varepsilon(\gamma, C) \\
\mathrm{y}=1.793 \mathrm{r}\end{array}$} & \multirow{2}{*}{$\zeta_{1}(\gamma)$} & \multicolumn{2}{|c|}{$\varepsilon(\gamma, C)$} & \multirow{2}{*}{$\zeta_{2}(\gamma)$} & \multicolumn{2}{|c|}{$\varepsilon(\gamma, \mathrm{C})$} & \multirow{2}{*}{$\zeta_{3}(\gamma)$} & \multirow{2}{*}{$\zeta_{1}(\gamma)$} & \multirow{2}{*}{$\sum_{j=1}^{2} \zeta_{j}(\gamma)$} & \multirow{2}{*}{$\sum_{j=1}^{3} \zeta_{j}(\gamma)$} \\
\hline & & & $y=3.105 r$ & $y=3.586 r$ & & $y=4.74 \pi$ & $y=5.379 r$ & & & & \\
\hline 2 & 0.1986 & 1.192 & 0.0559 & 0.0411 & 0.582 & 0.023 & 0.0177 & 0.382 & 1.192 & 1.774 & 2.16 \\
\hline 3 & 0.125 & 0.75 & 0.016 & 0.0099 & 0.155 & 0.0041 & 0.0027 & 0.065 & 0.75 & 0.905 & 0.97 \\
\hline 4 & 0.0935 & 0.561 & 0.005 & 0.0026 & 0.046 & 0.0008 & 0.0005 & 0.013 & 0.561 & 0.607 & 0.62 \\
\hline
\end{tabular}

where $\xi_{l}(\lambda)$ is given in (13). Consider rewriting (13) (after neglecting $F_{l}(\lambda)$ and $\left.\sum_{\substack{l=1 \\ l \neq 1}}^{L} I_{i, l,}\right)$ as 


$$
\xi_{l}(\lambda)=s_{l}+n_{l}+j_{l},
$$

where

$$
\begin{aligned}
& s_{l} \triangleq \sqrt{2 P} A T_{b} b_{l}^{(\lambda)}, \\
& n_{l} \triangleq D_{l}(\lambda)+N_{l}(\lambda)+\sum_{\substack{k=1 \\
k \neq i}}^{K} \sum_{l=1}^{L} I_{k, l} \hat{i}+\sum_{k=K+1}^{C K} \sum_{l=1}^{L} W_{k, l, i},
\end{aligned}
$$

and

$$
j_{l} \triangleq J_{l}(\lambda)
$$

Also, define

$$
\begin{gathered}
S_{T} \triangleq \sum_{l=1}^{U} s_{l}, \\
N_{T} \triangleq \sum_{l=1}^{U} n_{l}
\end{gathered}
$$

and

$$
J_{T} \triangleq \sum_{l=1}^{U} j_{l}
$$

Note that, following the same arguments as in [7], conditioned on $b_{t}^{(\lambda)}$ and $a_{i}(t), N_{T}$ is asymptotically normal as $K \rightarrow \infty$, with zero conditional mean and conditional variance approximately given by

$$
\begin{aligned}
& \operatorname{var}\left(N_{T}\right) \triangleq \sigma_{T}^{2}=U\left\{N_{0} T_{b} \sum_{m=-M}^{M} \alpha_{m}^{2}+P \rho T_{b}^{2}\right. \\
& \left.+P\left(A^{2}+2 \rho\right) T_{b}^{2} \frac{[1+\zeta(\gamma)] K L-L}{3 N} \sum_{m=-M}^{M}\left(2 \alpha_{m}^{2}+\alpha_{m} \alpha_{m+1}\right)\right\}(36)
\end{aligned}
$$

Therefore, we have for the conditional bit error rate, assuming $b_{i}^{(\lambda)}=+1$,

$$
P\left(e \mid a_{i}(t), J_{T}\right)=\phi\left[-\frac{S_{T}+J_{T}}{\sigma_{T}}\right],
$$

where

$$
\phi(x) \triangleq \frac{1}{\sqrt{2 \pi}} \int_{-\infty}^{x} \exp \left[-y^{2} / 2\right] d y .
$$

Now let us consider $J_{T}$. For future use, let us tie the processing gain, $N$, and number of CDMA users, $K$, together, in the sense of letting $N=N(K)$ in such a manner that $N$ increases monotonically with $K$. This can be justified physically by noting that any CDMA system must increase its processing gain as the number of users increases in order to maintain a satisfactory performance. However, the consequence of increasing processing gain is that either RF bandwidth increases or information rate decreases (or both). For the purposes of this paper, we assume $T_{b}$ is constant and that $T_{c}$ decreases as $N$ increases (i.e., we assume constant information rate but we allow the RF bandwidth to increase). For simplicity, assume that there are an integer number of bits of narrowband waveform in $T_{b}$ seconds, and ignore any timing offset between the bits of the BPSK signal and the bits of the reference CDMA signal. From (17), it is straightforward to show that

$$
\begin{aligned}
j_{l}=J_{l}(\lambda) & =\sqrt{2 J} \sum_{m=-M}^{M} \alpha_{m} \sum_{j=0}^{N-1} a_{j+m}^{(i)} d_{[p j]} \\
& \cdot\left[\frac{\sin \left[2 \pi \Delta\left(\lambda T_{b}+(j+1) T_{c}\right)-2 \pi \Delta m T_{c}+\hat{\theta}_{l}\right]}{2 \pi \Delta}\right. \\
& \left.-\frac{\sin \left[2 \pi \Delta\left(\lambda T_{b}+j T_{c}\right)-2 \pi \Delta m T_{c}+\hat{\theta}_{l}\right]}{2 \pi \Delta}\right],
\end{aligned}
$$

where $\left\{d_{j}\right\}$ is the data sequence of the narrowband BPSK waveform, $[x]$ is the largest integer less than $x$, $p \triangleq T_{c} / T_{j}=N_{1} / N$, and $N_{1}$ is the number of bits of the narrowband BPSK signal per bit of any of the CDMA waveforms. If we assume $\Delta T_{b}=N q$ is an integer, where $q \triangleq \Delta T_{c}$, we can ignore the term $\lambda T_{b}$ in the arguments of the sine functions. Further, if we define

$\beta\left(j, m, \hat{\theta}_{l}\right) \triangleq$

$$
\frac{\sin \left[2 \pi q(j+1-m)+\hat{\theta}_{l}\right]-\sin \left[2 \pi q(j-m)+\hat{\theta}_{l}\right]}{2 \pi q},
$$

then

$$
j_{l}=\sqrt{2 J} T_{c} \sum_{j=0}^{N-1} x_{j}
$$

where

$$
x_{j} \triangleq \sum_{m=-M}^{M} \alpha_{m} a_{j+m}^{(i)} d_{[p j]} \beta\left(j, m, \hat{\theta}_{l}\right) .
$$

Recall that

$$
\hat{\theta}_{l}=\theta+\phi_{i l} .
$$

Conditioned on $\theta+\phi_{i l}, x_{j}$ is a function of $d_{[p j]}$ and $\left\{a_{j-M}^{(i)}, \cdots, a_{j+M}^{(i)}\right\}$. It can be shown that the sequence $\left\{x_{j}\right\}$ is a $2 \mathrm{M}$ - dependent sequence ([12]). For example, consider the simple case of $\Delta=0, M=1$ and $1 / p=\frac{N}{N_{1}}=5$. Then, assuming $a_{j}^{(i)}=d_{j}=0$ for $j<0$, we have the following sequence:

$$
\begin{aligned}
& x_{0}=\alpha_{0} d_{0} a_{0}+\alpha_{1} d_{0} a_{1} \\
& x_{1}=\alpha_{-1} d_{0} a_{0}+\alpha_{0} d_{0} a_{1}+\alpha_{1} d_{0} a_{2} \\
& x_{2}=\alpha_{-1} d_{0} a_{1}+\alpha_{0} d_{0} a_{2}+\alpha_{1} d_{0} a_{3} \\
& x_{3}=\alpha_{-1} d_{0} a_{2}+\alpha_{0} \\
& x_{4}=\alpha_{-1} d_{0} a_{3}+\alpha_{0} \\
& x_{5}=\alpha_{-1} d_{1} a_{4}+\alpha_{0} \\
& x_{6}=\alpha_{-1} d_{1} a_{5}+\alpha_{0} \\
& x_{7}=\alpha_{-1} d_{1} a_{6}+\alpha_{0}
\end{aligned}
$$

Note that in the sequence $\left(x_{0}, x_{1}, x_{2}, \cdots\right\}$, if any $2 M=2$ consecutive $x_{i}^{\prime} s$ are removed, the remaining two subse- 
quences (the one to the left of the removed $x_{i}$ 's and the one to the right) are statistically independent.

Further, if $2 \pi q=\frac{k_{1} \pi}{k_{2}}$, where $k_{1}$ and $k_{2}$ are integers, then as $N \rightarrow \infty$, the sequence $\left\{x_{j}\right\}$ satisfies the conditions of the Hoeffding-Robbins version of the central limit theorem for dependent random variables ([12]). To illustrate this, consider again the above example. From [12], for a sum of $m$ dependent random variables to be asymptotically normal, it is necessary that

$$
\lim _{P \rightarrow \infty} \frac{1}{P} \sum_{h=1}^{P} A_{i+k}=A
$$

exist uniformly for all $i$, where

$$
A_{i} \triangleq 2 \sum_{j=0}^{m-1} \operatorname{cov}\left[x_{i+j}, x_{i+m}\right\}+\operatorname{var}\left(x_{i+m}\right) \text {. }
$$

In our example, $m=2 M=2$ and

$$
\operatorname{var}\left(x_{i}\right)=\sum_{j=-M}^{M} \alpha_{j}^{2}
$$

for all $i \geq 1$ (i.e., the variance term is independent of $i$ as long as $i \geq 1$ ). The covariance term can be shown to take on one of three possible values as long as $i>2$.

$$
\begin{aligned}
\sum_{j=0}^{l} \operatorname{cov}\left\{x_{i+j},\right. & \left.x_{i+2}\right\}= \\
& \begin{cases}0, & i=3+5 k \\
\alpha_{-1} \alpha_{0}+\alpha_{0} \alpha_{1}, & i=4+5 k \\
\alpha_{-1} \alpha_{1}+\alpha_{-1} \alpha_{0}+\alpha_{0} \alpha_{1}, & \text { otherwise }\end{cases}
\end{aligned}
$$

where $k=1,2,3, \cdots$. Since the variance term is independent of $i$ for $i \geq 1$, and the sum of covariances is periodic in $i$ for $i \geq 2$, it can be seen that $\frac{1}{P} \sum_{h=1}^{P} A_{i+h}$ equals the same value, independent of $i$, as $P \rightarrow \infty$.

If we call the conditional variance of $j_{l}$, conditioned on $\hat{\theta}_{l}, \sigma_{j}^{2}\left(\hat{\theta}_{l}\right)$, then

$$
\begin{aligned}
\sigma_{j}^{2}\left(\hat{\theta}_{l}\right) & =\left(2 J T_{b}^{2} / N^{2}\right) \sum_{m_{2}=-M}^{M} \sum_{m_{2}=-M}^{M} \alpha_{m_{1}} \alpha_{m_{2}} \\
& \cdot \sum_{j_{1}=0}^{N-1} \sum_{j_{2}=0}^{N-1} \beta\left(j_{1}, m_{1}, \hat{\theta}_{l}\right) \beta\left(j_{2}, m_{2}, \hat{\theta}_{l}\right) \\
& \cdot \delta\left(j_{1}+m_{1}, j_{2}+m_{2}\right) \delta\left(\left[p j_{1}\right],\left[p j_{2}\right]\right)
\end{aligned}
$$

where $\delta(i, j)$ is the Kronecker delta function. Further, since $j_{l_{1}}$ is uncorrelated with $j_{l_{2}}$ for $l_{1} \neq l_{2}$, the variance of $J_{T}$ is $\sigma_{J}^{2}\left(\bar{\phi}_{i}\right) \triangleq \sum_{l=1}^{U} \sigma_{j}^{2}\left(\hat{\theta}_{l}\right)$, where $\bar{\phi}_{i} \Delta\left[\hat{\theta}_{1}, \hat{\theta}_{2}, \cdots, \hat{\theta}_{U}\right\}$.

Finally, to obtain our approximation to the average BER, consider the following: From (37), we must average the conditional error rate over $a_{i}(t)$ and $J_{T}$. However, from (36), $\sigma_{T}^{2}$ is not a function of the $\left[a_{j}^{(i)}\right]$, and from $(48), \sigma_{j}^{2}\left(\hat{\theta}_{l}\right)$ is only a function of the random phase $\hat{\theta}_{l}$. Therefore,

$$
\begin{gathered}
P\left(e \mid a_{i}(t), J_{T}\right)=P\left(e \mid \bar{\phi}_{i}\right)=\frac{1}{\sqrt{2 \pi} \sigma_{J}\left(\bar{\phi}_{i}\right)} \int_{-\infty}^{\infty} \phi\left[-\frac{S_{T}+J_{T}}{\sigma_{T}}\right) \\
\cdot \exp \left\{-J_{T}^{2} / 2 \sigma_{J}^{2}\left(\bar{\phi}_{i}\right)\right\} d J_{T}=\phi\left[-\frac{S_{T}}{\sqrt{\sigma_{J}^{2}\left(\bar{\phi}_{i}\right)+\sigma_{T}^{2}}}\right),
\end{gathered}
$$

and

$$
P_{e}=E_{\bar{i}}\left\{P\left(e \mid \bar{\phi}_{i}\right)\right\}
$$

where $E_{\bar{\phi}}[\cdot]$ means expectation over the random vector $\bar{\phi}_{i}$. The total signal-to-noise ratio, conditioned on $\bar{\phi}_{i}$, is approximately given by

$$
\begin{aligned}
& S N R\left(\bar{\phi}_{i}\right) \triangleq \frac{1}{2} \frac{S_{T}^{2}}{\sigma_{T}^{2}+\sigma_{J}^{2}}=U\left\{\left[H \frac{E_{b}}{N_{0}}\right]^{-1} \sum_{m=-M}^{M} \alpha_{m}^{2}\right. \\
&+ \frac{1}{H}+\left(1+\frac{1}{H}\right) \frac{[1+\zeta(\gamma)] K L-L}{3 N} \sum_{m=-M}^{M}\left(2 \alpha_{m}^{2}+\alpha_{m} \alpha_{m+1}\right) \\
&+ \frac{J / S}{N^{2}} \cdot \frac{2}{U} \sum_{l=1}^{U}\left[\sum_{m_{1}=-M}^{M} \sum_{m_{2}=-M}^{M} \alpha_{m_{1}} \alpha_{m_{2}} \sum_{j_{2}=0}^{N-1} \sum_{j_{2}=0}^{N-1}\right. \\
& \cdot \beta\left(j_{1}, m_{1}, \hat{\theta}_{l}\right) \beta\left(j_{2}, m_{2}, \hat{\theta}_{l}\right) \\
&\left.\left.\cdot \delta\left(j_{1}+m_{1}, j_{2}+m_{2}\right) \delta\left(\left[p j_{1}\right],\left[p j_{2}\right]\right)\right]\right\}^{-1},
\end{aligned}
$$

where $\bar{E}_{b}=2 P \rho T_{b}$ is the average energy per coded bit, $J / S=J /\left(P A^{2}\right)$ denotes the interference power-to -useful signal power ratio, and $H=A^{2} /(2 \rho)$.

\section{B. Channel Coding}

We are interested in the performance of two simple block codes used in conjunction with the diversity. These are the BCH $(15,7)$ code and the Golay $(23,12)$ code. The former corrects two channel errors, while the latter corrects the three channel errors in each codeword. Assuming perfect interleaving, (i.e., ignoring the correlation introduced by both the suppression filter and the fading channel), the resulting bit error rate (BER) after hard decision decoding is approximately given by [13]

$$
P_{b} \approx \frac{1}{n} \sum_{i=1+1}^{n} i\left[\begin{array}{l}
n \\
i
\end{array}\right)\left(P_{e}\right)^{i}\left(1-P_{e}\right)^{n-i},
$$

where $n$ is the number of bits in a codeword and $t$ denotes the number of errors that the code can correct.

\section{Determination of the Suppression (Wiener) Filter Coefficients}

The coefficients of the suppression filter can be determined by solving the following Wiener-Hopf equation:

$$
\sum_{\substack{m=-M \\ m=0}}^{M} \alpha_{m} \hat{\rho}\left[(n-m) T_{c}\right]+\hat{\rho}\left(n T_{c}\right)=0,
$$


where $n=-M, \cdots, M, n \neq 0$, and $\hat{\rho}(\cdot)$ is the low-pass version (normalized to the received useful power $P A^{2}$ of the reference user) of the autocorrelation function of the input signal to the suppression filter and is given by

$$
\begin{aligned}
& \hat{\rho}\left(l T_{c}\right) \\
& \quad \begin{cases}(1+1 / H)[1+\zeta(\gamma)] K L+2 N\left[\bar{E}_{b} / N_{0}\right]^{-1}+J / S, & l=0 \\
(J / S)(1-|l| p) \cos (2 \pi l q), & |l| \leq[1 / p] \\
0, & |l| \geq[1 / p]\end{cases}
\end{aligned}
$$

where $p$ and $q$ are given by (6) and (7), respectively.

\section{NUMERICAL RESULTS}

In this section, we present numerical results for the BER of a CDMA system in the presence of the narrowband interference. Unless noted otherwise, it is assumed that the ratio of the interference bandwidth to the spread spectrum bandwidth is $10 \%(\mathrm{p}=0.1)$, the ratio of the offset of the interference carrier frequency to half of the spread spectrum bandwidth is $20 \%$ ( $q=0.2$, the ratio of the specular component power to the fading component power, $H$, is $7 \mathrm{~dB}$, and the processing gain, $N$, is 255 . Note that where the BER is plotted as a function of the ratio $\bar{E}_{i} / N_{0}$, this corresponds to the energy-per -bit to noise spectral density of an uncoded system.

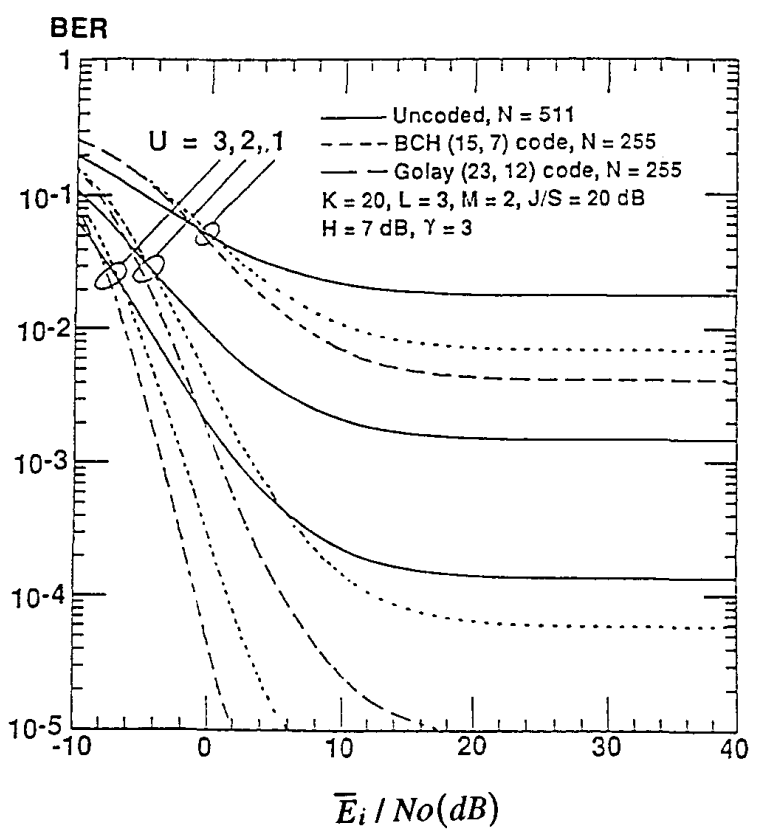

Fig. 4. The BER performance of DS-CDMA overlay system with diversity plus coding.

In Fig. 4, the decoded BER for $\mathrm{H}=7 \mathrm{~dB}$ is shown. Also, the uncoded BER is plotted in order to allow for comparison. Note that the uncoded and coded systems are compared for the same spread spectrum bandwidth $B=2 N /\left(T_{i} m / n\right)$. In our example, $\mathrm{N}=255$ for the coded systems, whereas $\mathrm{N}=511$ for the uncoded system. As expected, both the uncoded and coded BER decreases as the order, $U$, of diversity increases. The performance advantage obtained by using diversity becomes larger as the error correcting capability of the code increases for the same spread bandwidth.

In order to investigate the relative effect of the narrowband interference on the performance of the CDMA system with respect to the multiple-access and adjacent-cell interference, Fig. 5 illustrates the asymptotic $\left(\bar{E}_{i} / N_{0} \rightarrow \infty\right)$



Fig. 5. The asymptotic $\left(\bar{E}_{i} / N_{0} \rightarrow \infty\right)$ decoded BER of DS-CDMA overlay system as a function of $(J / S) /[(1+\zeta(\gamma)) K L]$.

BER of the CDMA system both with or without the suppression filter as a function of $(J / S) /[(1+\zeta(\gamma)) K L]$. Note that the ratio of the narrowband interference to the multiple-access and adjacent-cell interference can be roughly represented by $(J / S) /[(1+\zeta(\gamma)) K L]$. As expected, the BERs with or without suppression filters increase as $J / S$ increases. When $J / S$ is small, the multiple-access and adjacent-cell interference dominates; in this case, the BERs of the systems both with and without a suppression filter are very close, so that the suppression filter is unnecessary. When $J / S$ is large, the narrowband interference is on the order of the multiple-access and adjacent-cell interference; in this case, the system without a suppression filter degrades significantly, whereas the system is much more tolerant of the interference when the suppression filter is present.

In Fig. 6, the asymptotic BER of the system with and without the suppression filter is shown as a function of the ratio $(p)$ of the interference bandwidth to the spread spectrum bandwidth. It is seen from the figure that the CDMA system with a double-sided Wiener filter can suppress the nar- 
rowband interference very effectively (assuming, of course, that $p$ does not become too large).

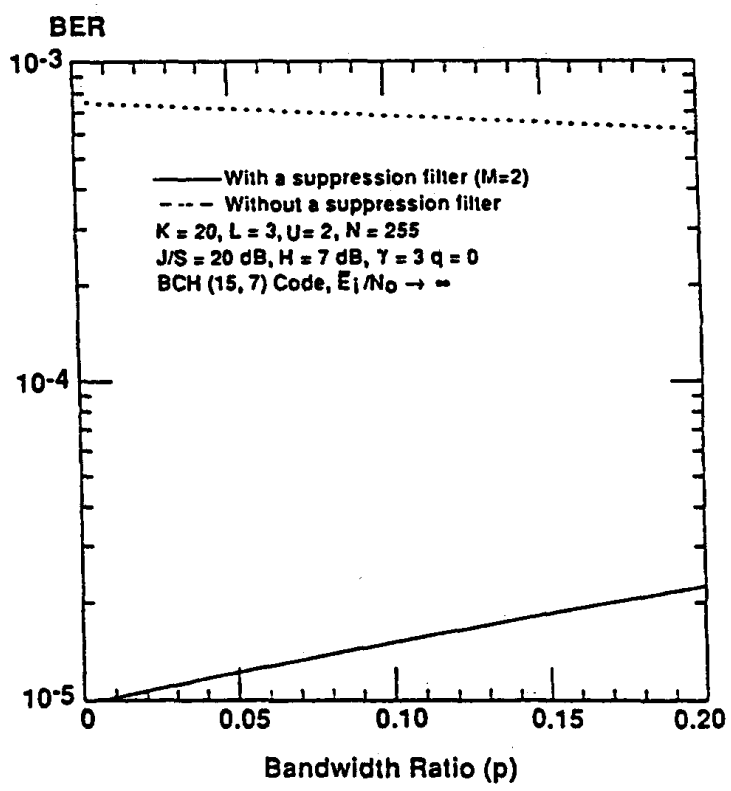

Fig. 6. The asymptotic $\left(\bar{E}_{i} / N_{0} \leftarrow \infty\right)$ BER of DS-CDMA system with and without suppression filters as a function of the bandwidth ratio $(p)$ of the interference $\mathrm{BW}$ to the spread spectrum $\mathrm{BW}$.

Fig. 7 illustrates the asymptotic BER for the system employing the suppression filter as a function of the number

Table II $(K=20, L=3 \mathrm{U}=2, \mathrm{~N}=255, \mathrm{M}=2, \mathrm{q}=0, \mathrm{H}=7 \mathrm{~dB}, \gamma=3, \mathrm{~J} / \mathrm{S}=20 \mathrm{~dB}, \mathrm{BCH}(15,7)$ code) of taps on each side, $M$. It is seen from the figure that the BER decreases first when $M=1$ and then keeps a steady value with increasing $M$. For $M \geq 1$, the narrow-band interference is reduced by the suppression filter, and increasing $M$ beyond 1 does not noticeably reduce the narrowband interfer-

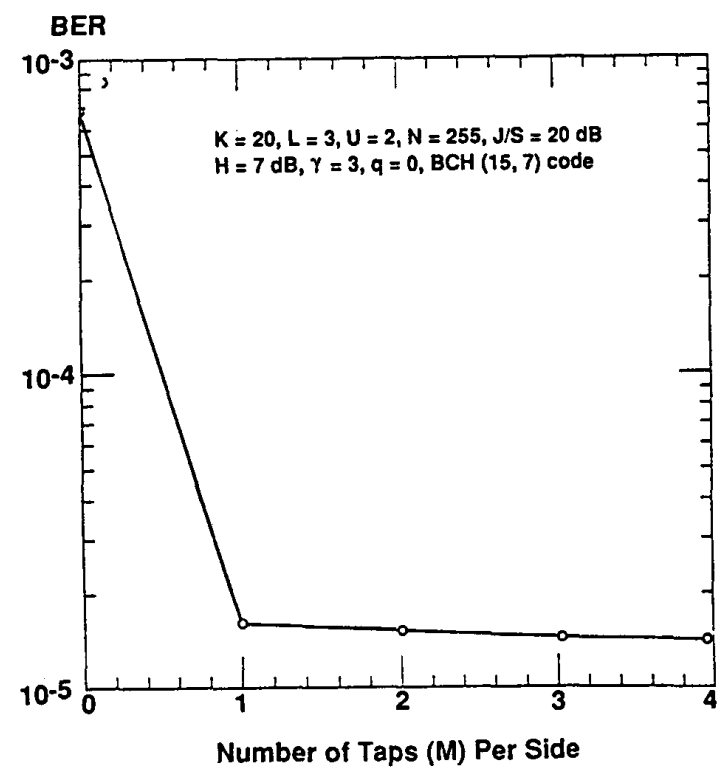

Fig. 7. The asymptotic $\left(\bar{E}_{\mathrm{i}} / N_{0} \rightarrow \infty\right)$ BER of DS-CDMA systems with a suppression filter as a function of number of taps on each side.

\begin{tabular}{|c|c|c|c|c|c|c|c|c|c|c|c|}
\hline \multirow{2}{*}{$\begin{array}{l}\text { Number of } \\
\text { taps per } \\
\text { side }(\mathrm{M})\end{array}$} & \multicolumn{9}{|c|}{ Coefficients $\left(\alpha_{m}\right)$} & \multirow{2}{*}{$\mathbf{Q}$} & \multirow{2}{*}{ w } \\
\hline & $\alpha_{-4}$ & $\alpha_{-3}$ & $\alpha_{-2}$ & $\alpha_{-1}$ & $\alpha_{0}$ & $\alpha_{1}$ & $\alpha_{2}$ & $\alpha_{3}$ & $\alpha_{4}$ & & \\
\hline 0 & $\mathbf{0}$ & $\mathbf{0}$ & $\mathbf{0}$ & $\mathbf{0}$ & 1 & $\mathbf{0}$ & $\mathbf{0}$ & 0 & 0 & 0.379 & 0.36 \\
\hline 1 & 0 & 0 & 0 & -0.302 & 1 & -0.302 & 0 & 0 & 0 & 0.079 & 0.33 \\
\hline 2 & 0 & $\mathbf{0}$ & -0.165 & -0.213 & 1 & -0.213 & -0.165 & 0 & 0 & 0.056 & 0.35 \\
\hline 3 & 0 & -0.1 & -0.131 & -0.185 & 1 & -0.185 & -0.131 & -0.1 & 0 & 0.053 & 0.35 \\
\hline 4 & -0.063 & -0.083 & -0.119 & -0.174 & 1 & -0.174 & -0.119 & -0.083 & -0.063 & 0.054 & 0.35 \\
\hline \multicolumn{12}{|c|}{$\mathrm{Q}=\frac{2 \mathrm{~J} / \mathrm{S}}{\mathrm{N}^{2}} \sum_{\mathrm{m}_{1}=-\mathrm{M}}^{\mathrm{M}} \sum_{\mathrm{m}_{2}=-M}^{M} \alpha_{\mathrm{m}_{1}} \alpha_{\mathrm{m}_{2}} \sum_{\mathrm{j}_{1}=0}^{\mathrm{N}-1} \sum_{\mathrm{j}_{2}=0}^{N-1} \mathrm{E}_{\Phi_{1}}\left[\beta\left(\mathrm{j}_{1}, \mathrm{~m}_{1}, \hat{\theta}_{l}\right) \beta\left(\mathrm{j}_{2}, \mathrm{~m}_{2}, \hat{\theta}_{l}\right)\right] \delta\left(\mathrm{j}_{1}+\mathrm{m}_{1}, \mathrm{j}_{2}+\mathrm{m}_{2}\right) \delta\left[[\mathrm{pj}],\left[\mathrm{pj} j_{2}\right]\right]$} \\
\hline
\end{tabular}

Table II. Tap Coefficients and Their Effects on the Interference 
ence, because $\hat{\rho}\left(T_{c}\right)$ and $\hat{\rho}\left(-T_{c}\right)$ are almost identical to $\hat{\rho}(0)$ in (54). Thus, the suppression filter with $M=1$ is sufficient to reject the interference in this case (small $p$ and $q=0$ ), and more taps than one on each side are unnecessary. Table II shows the tap coefficients of the suppression filters for different numbers of taps each side $(M=0,1,2,3)$. As shown in Table II, for $M=0, Q=W$, where $Q$ roughly represents the effect of the narrowband interference and $W$ represents the effect of the multiple access interference; in this case, both the narrowband interference and multiple-access interference have comparable effects. However, for $M \geq 1$, $Q \ll W$ and $W$ does not vary very much. Therefore, we conclude that when the carrier frequencies of the narrowband interference and CDMA signals are the same, the suppression filter with three total taps is sufficient to reduce the narrowband interference, and increasing the number of total taps beyond three is not necessary.

Fig. 8 illustrates the BER performance of the systems with the suppression filter as a function of the ratio $(q)$ of the

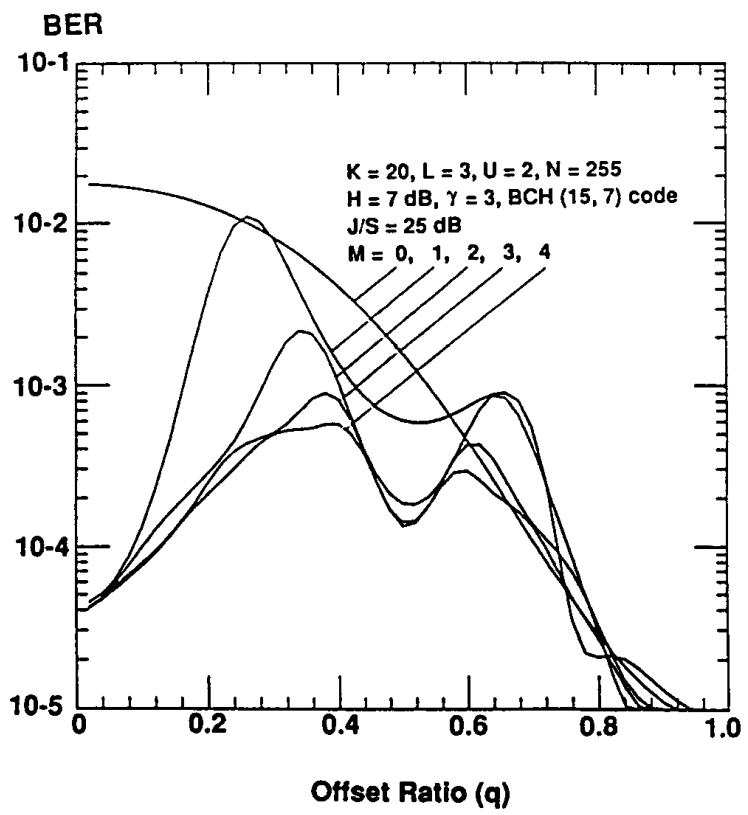

Fig. 8. The asymptotic $\left(\bar{E}_{\mathrm{i}} / N_{0} \rightarrow \infty\right)$ BER of the system with doublesided filters as a function of the offset ratio $(q)$ of the interference carrier frequency to the half spread spectrum $\mathrm{BW}$.

offset of the interference carrier frequency to the half spread spectrum bandwidth for different number of the taps on each side. It is seen that when $q$ is very small, the BERs of the system with double-sided filters for $M \geq 1$ are almost identi$\mathrm{cal}$, as was the case in Fig. 7. However, the BER performance improves as $M$ increases when $\mathrm{q}$ increases, and the BER for large $M$ is also more robust to a change in $q$. Also, for $q \approx 0.25$, the BERs of the systems with the double-sided suppression filter and without the suppression filter are almost identical, because the low-pass version of the autocorrelation of the interference is zero, (i.e., $\rho_{j}\left(l T_{c}\right)=0$ for $l \neq 0$ ), so that all off-center tap coefficients are zero.
In Fig. 9, the asymptotic BER of the DS-CDMA system with a suppression filter is plotted as a function of the number

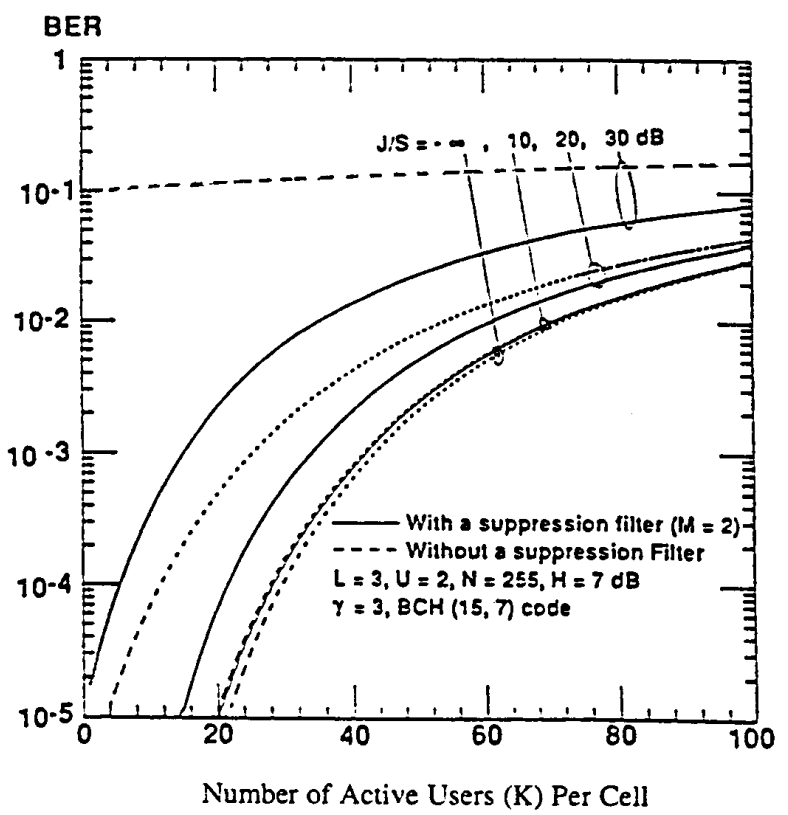

Fig. 9. The asymptotic decoded BER of DS-CDMA overlay systems as a function of the number of active users per cell.

of active users, $\mathrm{K}$, for various values of J/S. Also, the BER of the system without the suppression filter is shown for comparison. It is seen that at large $\mathrm{J} / \mathrm{S}$, for a given BER, the system with the suppression filter can support many more users

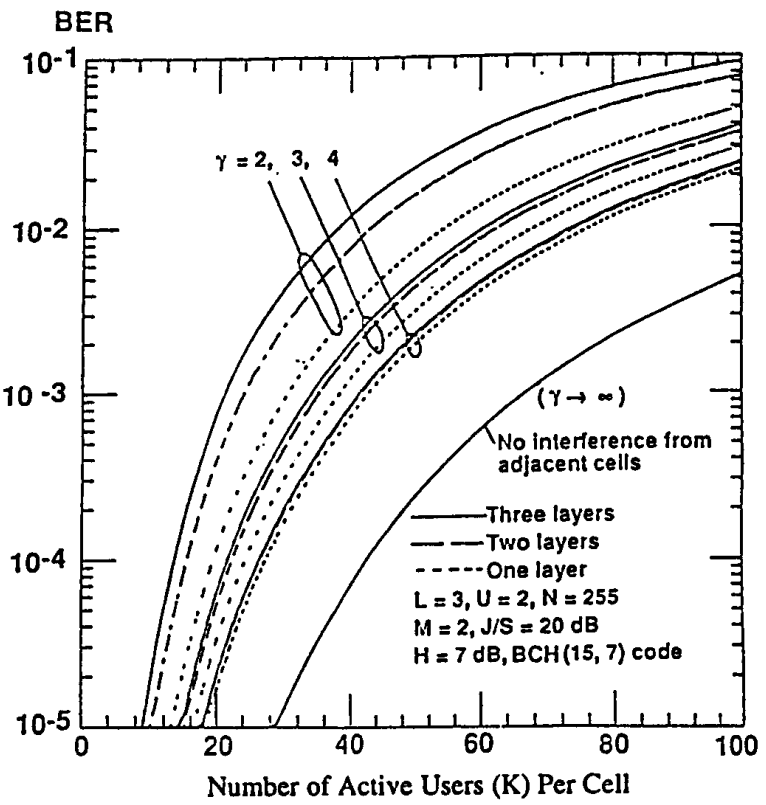

Fig. 10. The asymptotic decoded BER of DS-CDMA overlay system as a function of active users $(K)$ per cell for various values of the propagation loss exponent $(\gamma)$. 
than can the system without the suppression filter. When the interference is such that $J / S \leq 10 d B$, the multiple-access and adjacent-cell interference dominates so that the suppression filter is not needed, as was seen in Fig. 5.

Fig. 10 illustrates the asymptotic BER of the CDMA system versus $\mathrm{K}$, the number of active users per cell, for various values of the propagation loss exponent, $\gamma,(\gamma=2,3,4)$. Also, the BER in the absence of adjacent cell interference (i.e., $\gamma \rightarrow \infty$ ) is shown for comparison. In order to show the effect of the adjacent-cell interference on the performance of the system for different values of $\gamma$, a single layer of cells, two layers of cells and three layers of cells are considered. It is clear from this figure that when $\gamma=2$, the BER computed by accounting for only a single layer of cells is too optimistic; alternately, if $\gamma \geq 3$, the difference in BER performance between accounting for only a single layer and accounting for more layers is insignificant. Further, for a given $\gamma$, the gap in the BER between two and three layers is much less than that between one and two layers. Indeed, these results can be seen from the $\zeta_{j}(\gamma)$ entries of Table I, and are consistent with the results presented in [6].

\section{CONCLUSIONS}

An approximate technique has been described for determining the performance of a DS-CDMA system operating over a multipath Rician fading channel and sharing common spectrum with various narrowband waveforms. It was shown that

(1) When the narrowband interference power-to-signal power ratio $(J / S)$ is small with respect to the multipleaccess and adjacent-cell interference, the suppression filter is unnecessary, whereas when $J / S$ is on the order of the multiple-access and adjacent-cell interference, the suppression filter provides significant enhancement in performance;

(2) When the ratio $(q)$ of the offset of the interference carrier frequency to half of the spread spectrum bandwidth is very small, that is, when the interference carrier frequency is very close to the spread spectrum carrier frequency, spread spectrum systems with double-sided suppression filters only need three total taps. However, the double-sided filter with a large number of taps (i.e., $M>1$ ) is preferable when the ratio is large.

(3) For large narrowband interference, the system with a suppression filter can support many more users than can the system without a suppression filter;

(4) As is well known, the number of users supported by the CDMA overlay system increases as the propagation loss exponent $(\gamma)$ increases. For typical exponents, three layers of cells are sufficient for considering the adjacent cell interference.

\section{REFERENCES}

[1] R. L. Pickholtz, L. B. Milstein and D. L. Schilling, "Spread spectrum for mobile communications," IEEE Trans. Vehicular Tech., vol. VT40, pp. 313-322, May 1991.

[2] D. L. Schilling, L. B. Milstein, R. L. Pickholtz and F. Miller, "CDMA for personal communications networks," Proc. of MIL COM'90, pp. 28.2.1-28.2.4, October 1990.

[3] T. S. Rappaport and L. B. Milstein, "Effects of path loss and fringe user distribution on CDMA cellular frequency reuse efficiency," Proc. of GLOBECOM'90, pp. 404.6.1-404.6.7, December 1990.

[4] R. J. Bultitude and G. K. Bedal, "Propagation characteristics on microcellular urban mobile radio channels at $910 \mathrm{MHz}$, IEEE J. Select. Areas Comnun., vol. SAC-7, pp.31-39, Jan. 1989.

[5] G. R. Cooper and R. W. Nettleton, "A spread-spectrum technique for high-capacity mobile communications," IEEE Trans. Vehicular Tech., vol. VT-27, pp.264-275, Nov. 1978.

[6] L. B. Mi]stein, T. S. Rappaport and R. Barghouti, "Performance evaluation for cellular CDMA," IEEE Joumal on Select. Areas in Commun., vol. SAC-10, pp. 680-689, May 1992.

[7] J. Wang, M. Moeneclaey and L. B. Milstein, "DS-CDMA with predetection diversity for indoor radio communications," IEEE Trans. Comm., vol. COM-42, pp. 1929-1938, April 1994.

[8] M. B. Pursley, "Performance evaluation for phase-coded spread spectrum multiple access communications, Part I: system analysis," IEEE Trans. on Commun, vol. Com-25, pp. 295-299, Aug. 1977.

[9] J. S. Lehnert and M. B. Pursley, "Error probabilities for binary direct sequence spread spectrum multiple access communications with random signature sequences," IEEE Trans. Commun, vol. COM-35, pp. 87-98, Jan. 1987.

[10] R. K. Morrow, Jr. and J. S. Lehnert, " Bit-to-bit error dependence in sloted DS/SSMA packet systems with random signature sequences," IEEE Trans. Commun., vol. COM-37, pp. 1052-1061, Oct. 1989.

[11] M. B. Pursley, "The role of spread spectrum in packet radio networks," Proceedings of IEEE, vol. 75, pp. 116-134, Jan. 1987.

[12] D. A. S. Fraser, Nonparametric Methods in Statistics, Wiley 1957, pp. 215-218.

[13] E. R. Berlekamp, "The technology of error-correcting codes," Proceedings of IEEE, vol. 68, pp. 564-593, May 1980.

[14] L. B. Milstein, "Interference rejection techniques in spread spectrum communications," Proceedings of IEEE, vol. 76, pp. 657-671, June 1988.

Jiangzhou Wang (M'91-SM'94) was born in Hubei, China, on November 15, 1961. He received the B.S. and M.S. degrees from Xidian University, Xian, China, in 1983 and 1985, respectively, and the Ph.D. degree (with Greatest Distinction) from the University of Ghent, Belgium, in 1990, all in electrical engineering.

From December 1990 to June 1992, he was a Postdoctoral Fellow in the Department of Electrical and Computer Engineering, University of California at San Diego. Since July 1992, he has been a Senior System Engineer in the Digital Communications Division, Rockwell Corporation, Newport Beach, California, where he is currently working on the development and system design of wireless communications. He has contributed one pending US patent, entitled "Variable multithreshold detection for 0.3-GMSK," which can be applied to wireless systems, such as GSM and Mobitex.

Laurence B. Milstein (S'66-M'68-SM'75-F' 85) received the B.E.E. degree from the City College of New York, New York, NY, in 1964, and the M.S. and Ph.D. degrees in elestrical engineering from the Polytechnic Institute of Brooklyn, Brooklyn, NY, in 1966 and 1968, respectively.

From 1968 to 1974, he was employed by the Space and Communications Group of Hughes Aircraft Company, and from 1974 to 1976 he was a member of the Department of Electrical and Systems Engineering, Rensse- 
laer Polytechnic Institute, Troy, NY. Since 1976, he has been with the Department of Electrical and Computer Engineering, University of California at San Diego, La Jolla, CA, where he is a Professor and former Department Chairman, working in the area of digital communication theory with special emphasis on spread-spectrum communication systems. He has also been a consultant to both government and industry in the areas of radar and communications. He was an Associate Editor for Communication Theory for the IEEE Transactions on Communications, an Associate Editor for Book Reviews for the IEEE Transactions on Information Theory, and an Associate Technical Editor for the IEEE Communications Magazine, and is currently a Senior Editor for the IEEE Journal on Selected Areas in Communications.

He was the Vice President for Technical Affairs in 1990 and 1991 of the IEEE Communications Society, and is currently a member of the Board of Governors of both the IEEE Communications Society and the IEEE Information Theory Society. 